\title{
Prejuízos nutricionais e distúrbios no padrão de sono de trabalhadores da Enfermagem
}

\author{
Nutritional damages and disturbances in the sleep pattern of nursing workers
}

Prejuicios nutricionales y alteraciones en los patrones de sueño de los trabajadores de Enfermería

\author{
Martina Pafume Coelho', Olaine Oliveira Pinto', \\ Maria Carliana Mota', Cibele Aparecida Crispim' \\ ' Universidade Federal de Uberlândia, Faculdade de Medicina, \\ Programa de Pós-Graduação em Ciências da Saúde. Uberlândia-MG, Brasil.
}

\author{
Submissão: 01-11-2012 Aprovação: 29-08-2014
}

\section{RESUMO}

Este artigo apresenta uma revisão integrativa das publicações científicas da última década, que investigaram os hábitos de sono, a ingestão alimentar e o estado nutricional de profissionais de enfermagem. Foram analisados artigos publicados em periódicos nacionais e internacionais no período de 2002 a 2014, disponibilizados na base de dados PubMed/MEDLINE (USA National Library of Medicine), Lilacs / SciELO (Scientific Eletronic Library Online) e Google Acadêmico. Trinta e um artigos preencheram os critérios estabelecidos. Na análise destes estudos foi identificada elevada prevalência de sobrepeso e obesidade, além de uma modificação negativa nos hábitos alimentares, bem como prejuízos na dinâmica do sono dos profissionais da área de enfermagem.

Descritores: Enfermagem; Hábitos de Sono; Ingestão Alimentar; Estado Nutricional.

This article presents an integrative review of national and international scientific publications that investigate the sleep habits, the feed intake and nutritional status of nursing professionals. It was analyzed articles published in national and international journals in the period 2002 to 2014 and made available in the database PubMed / MEDLINE (USA National Library of Medicine), Lilacs / SciELO (Scientific Eletronic Library Online) and Google Scholar. Thirty one articles met the criteria. In the analysis of these studies it has been found a high prevalence of overweight and obesity, a negative change in the eating habits, as well as losses in the sleep patterns of nursing professionals.

Key words: Nursing; Sleep Habits; Dietary Intake; Nutritional Status.

\section{RESUMEN}

En este artículo se presenta una revisión integradora de las publicaciones científicas nacionales e internacionales que investigan los hábitos de sueño, el consumo de alimento y el estado nutricional de los profesionales de enfermería. Se analizaron los artículos publicados en revistas nacionales e internacionales en el período de 2002 a 2014, disponibles en la base de datos PubMed / MEDLINE (USA Biblioteca Nacional de Medicina), Lilacs / SciELO (Scientific Eletronic Library Online) y Google Scholar. Treinta y uno artículos cumplieron con los criterios de inclusión. En el análisis de estos estudios se encontró una alta prevalencia de sobrepeso y obesidad, un cambio negativo en los hábitos alimenticios, así como prejuicios en la dinámica del sueño de los profesionales de enfermería.

Palabras clave: Enfermería; Hábitos de Sueño; Ingesta Alimentaria; Estado Nutricional.

\section{AUTOR CORRESPONDENTE Martina Pafume Coelho E-mail: martina@psi.ufu.br}




\section{INTRODUÇÃO}

O trabalho em turnos surgiu após a organização das sociedades e se intensificou durante a Revolução Industrial e também com o advento da luz artificial, o que permitiu prolongar as atividades cotidianas, inclusive para o período noturno ${ }^{(1)}$. Com o desenvolvimento dos processos tecnológicos, mudanças econômicas e demográficas, bem como com o estreitamento e globalização das relações entre países, o trabalho em turnos se tornou imprescindível numa sociedade com ritmo de trabalho de 24 horas $^{(2)}$.

Existem diferentes tipos de trabalhadores que exercem suas atividades profissionais no esquema de turnos. Entre esses, destacam-se os trabalhadores envolvidos na prestação de serviços de saúde, como os profissionais da área de Enfermagem $^{(1)}$. Nesse caso, o trabalho em horários não convencionais e as longas jornadas são fonte de desgaste físico e emocional, que repercutem negativamente no estilo de vida, comprometendo, entre outros fatores, a qualidade do sono e o comportamento alimentar ${ }^{(3)}$.

Evidências atuais têm postulado que o número, a duração e a irregularidade de horários dos turnos de trabalho afetam de forma importante o padrão de sono ${ }^{(2,4-6)}$. Além disso, esse esquema laboral pode comprometer horários e frequência das refeições e as preferências alimentares destes profissionais ${ }^{(7)}$, deteriorando os hábitos alimentares ${ }^{(8-10)}$. Esses prejuízos no padrão de sono e nos hábitos alimentares, os quais podem estar intimamente interligados ${ }^{(10-11)}$, têm se mostrado capazes de predispor a obesidade e suas comorbidades associadas ${ }^{(10,12)}$. Estudos apontam que estado nutricional dos profissionais da Enfermagem pode sofrer alterações negativas, o que é demonstrado pela elevada prevalência de sobrepeso e obesidade nessa população ${ }^{(2-3,13)}$.

Deste modo, esse artigo tem como objetivo elaborar uma revisão integrativa da literatura, abordando a influência do trabalho em turnos sobre o perfil nutricional e o padrão de sono dos profissionais da área da Enfermagem que atuam no esquema de turnos, com especial ênfase na relação entre a privação do sono e os hábitos alimentares.

\section{METODOLOGIA}

Trata-se de um estudo do tipo revisão integrativa, um método de pesquisa que possibilita a síntese de estudos publicados e geram conclusões gerais a respeito de uma determinada área de pesquisa. Estudos de revisão integrativa têm como objetivo obter um entendimento relevante sobre um determinado fenômeno com base em estudos realizados anteriormente ${ }^{(14)}$. Esse método consiste em uma ampla análise da literatura o que contribui para discussões acerca de métodos e resultados de pesquisas, além de apontar lacunas do conhecimento que podem ser preenchidas com a realização de novos estudos ${ }^{(14-15)}$.

Este estudo foi baseado no método de Beyae e Nicoll ${ }^{(16)}$ incluindo as seguintes etapas: seleção do tema e da questão da pesquisa; estabelecimento de critérios para inclusão e exclusão de estudos; definição das informações gerais a serem extraídas dos estudos; avaliação dos estudos incluídos na revisão integrativa; interpretação dos resultados e apresentação da revisão.

Nesta revisão, formulou-se a seguinte questão norteadora: "O turno de trabalho pode influenciar a ingestão alimentar e/ ou o estado nutricional e/ou os hábitos de sono de profissionais da área da Enfermagem"?

O levantamento bibliográfico foi realizado por meio de consultas nas bases de dados PubMed / MEDLINE (USA National Library of Medicine), Lilacs / SciELO (Scientific Eletronic Library Online) e Google Acadêmico, no período de 2002 a 2014. Na base Pubmed / MEDLINE foi utilizada a associação dos descritores "nursing professional" e "shift work" com o descritor "sleep" e foram encontradas 12 publicações. Ao associar os descritores "nursing professional" e "shift work" com "food intake", "body weight" e "obesity", foi registrada uma ocorrência de publicação indexada. Assim, foi utilizado o descritor "shift work" associado aos descritores "food intake" e/ou "body weight" e/ou "obesity" e foram selecionadas apenas as publicações relacionadas aos profissionais da área de enfermagem. Nas bases Lilacs / SciELO e Google Acadêmico a busca foi realizada utilizando o descritor "enfermeiro" associado ao descritor "sono", pelo método integrado e foram encontradas seis publicações. Utilizou-se também o descritor "enfermeiro" associado aos descritores "ingestão alimentar" e/ou "peso corporal" e/ou "obesidade" e foram encontradas duas publicações.

Para compor a amostra foram utilizados os seguintes critérios: apresentar desfecho relacionado ao turno de trabalho e sua influência sobre a ingestão alimentar e/ou sobre o estado nutricional e/ou sobre os hábitos de sono de profissionais da área de enfermagem. Para análise desses critérios foi necessária a leitura de cada resumo das publicações encontradas visando identificar o panorama geral da pesquisa. Definindo-se os trabalhos selecionados, foram capturados os textos completos e sintetizados os resultados principais acerca do foco deste estudo (ingestão alimentar, estado nutricional e hábitos de sono de enfermeiros, técnicos e auxiliares de enfermagem que trabalham em instituições hospitalares).

Inicialmente, a amostra foi constituída de 42 publicações que atenderam aos critérios de busca. Após a leitura dos textos completos foram excluídos 11 estudos que, apesar de mencionarem as palavras-chave "enfermeiro" associado com "ingestão alimentar" ou "peso corporal" ou "obesidade" e suas devidas traduções para a língua inglesa ou espanhola, não aderiram aos objetivos dessa revisão.

\section{RESULTADOS}

Foram identificados 31 artigos que preencheram os critérios estabelecidos. As evidências foram provenientes, em sua maioria (29), de estudos de natureza quantitativa com desenho transversal e, também, de dois estudos de coorte. A prática clínica baseada em evidências tem como pilar o uso de resultados de pesquisas ${ }^{(17)}$. A qualidade da evidência é atribuída pela sua validade e relevância, portanto, antes de ser utilizada, deve ser avaliada quanto à acurácia, relevância e aplicabilidade em determinada situação ${ }^{(17)}$. 
A literatura tem caracterizado as evidências de forma hierárquica, assim, quanto mais bem delineada é a pesquisa, mais fortes são as evidências ${ }^{(18)}$, o importante é que o desenho das pesquisas utilizadas seja capaz de responder a questão norteadora $^{(18-19)}$. Estudos observacionais do tipo coorte possuem níveis de evidencia tipo IV e do tipo transversal, possuem nível de evidência do tipo $\mathrm{V}^{(19)}$. O grau de recomendação desses estudos é classificado como $\mathrm{B}^{(19)}$.

\section{Perfil nutricional de profissionais de enfermagem}

É atualmente reconhecido pela literatura que os profissionais da área da Enfermagem constituem uma população de risco para o ganho de peso de forma inadequada e que a prevalência de obesidade neste grupo é elevada. O Quadro 1 mostra estudos da literatura desenvolvidos em diferentes países que analisaram o estado nutricional de profissionais da área de enfermagem.

Em face destes números, algumas discussões são pertinentes, como a comparação da prevalência de sobrepeso e obesidade de profissionais da área da Enfermagem versus prevalência da população em geral, tendo em vista que os números populacionais se mostram muito elevados em todo o mundo. Neste sentido, um estudo de coorte conduzido na Austrália ${ }^{(19)}$ investigou o peso corporal de profissionais de enfermagem e encontrou prevalência de $61,87 \%$ de sobrepeso e obesidade (Índice de Massa Corporal (IMC) $\geq 25 \mathrm{~kg} / \mathrm{m}^{2}$ ). Esse achado representou uma prevalência de sobrepeso e de obesidade até $3,74 \%$ maior do que da população australiana.

No Brasil, de acordo com o Instituto Brasileiro de Geografia e Estatística (IBGE) (20) a prevalência de excesso de peso na população adulta é de, aproximadamente, 60\%. Alguns estudos brasileiros identificaram elevadas taxas do excesso de peso corporal nos profissionais da área de enfermagem. Em estudo transversal com profissionais de enfermagem ${ }^{(21)}$ encontrou-se prevalência de sobrepeso e de obesidade (IMC $\geq 25 \mathrm{~kg} / \mathrm{m}^{2}$ ) em 56,3\% da amostra. Outras pesquisas brasileiras $^{(1,22-24)}$ também verificaram a ocorrência de sobrepeso de obesidade em profissionais da área da Enfermagem $(p<0,05)$. Dessa maneira, podemos observar que as taxas verificadas entre profissionais da área da Enfermagem são, muitas vezes, superiores às apresentadas na população, o que sugere que o ganho de peso pode, ao menos em parte, ser influenciado pelas condições impostas pela atividade profissional.

Outro aspecto importante nessa questão é a presença de outros fatores de risco associados à obesidade entre profissionais da Enfermagem. Uma pesquisa conduzida na Coréia do Sul(25) identificou que a relação cintura-quadril (RCQ), um importante preditor das doenças cardiovasculares, crescia proporcionalmente ao aumento do número de anos trabalhados em turnos noturnos entre enfermeiros $(p<0,05)$. Entretanto, nesse estudo, o IMC não foi significativamente relacionado à duração do trabalho em turnos. No Brasil, identificou-se que a circunferência da cintura, que também prevê riscos de doenças cardiovasculares, estava acima dos valores limítrofes em $75 \%$ da amostra de profissionais de enfermagem ${ }^{(21)}$. Isso demonstra que esta modalidade laboral pode se relacionar com fatores de risco para outras patologias, como as doenças cardiovasculares.

Dentre os estudos selecionados na presente revisão, apenas um não relacionou o turno de trabalho ao aumento do peso corporal. Na Espanha, investigaram-se 207 profissionais de enfermagem do turno rotativo e fixo em hospitais ${ }^{(26)}$. Ao contrário dos demais estudos com profissionais dessa área ${ }^{(7,27-29)}$, observou-se que apenas 19\% dos participantes apresentavam sobrepeso e $4 \%$, obesidade. A média de IMC observada foi de $23,17 \mathrm{~kg} / \mathrm{m}^{2}$. De acordo com os autores, os resultados encontrados nos profissionais do turno rotativo noturno estão dentro da faixa recomendada para a população daquele país ${ }^{(26)}$.

Esses resultados sugerem que o trabalho em turnos pode alterar o peso corporal de profissionais da área de enfermagem. Estudos de seguimento, como os de modelo coorte, são fundamentais para avaliar os agentes causadores do excesso de peso entre estes profissionais e isolar possíveis fatores relacionados ao trabalho que levem ao excesso de peso.

Quadro 1 - Perfil nutricional de profissionais da área de enfermagem

\begin{tabular}{|c|c|c|c|}
\hline Autor(es), Ano, País & Delineamento & População & Principais resultados \\
\hline $\begin{array}{l}\text { Kim et al, } 2013 \\
\text { Coreia do Sul }\end{array}$ & Transversal & $\begin{array}{l}9.851 \\
\text { (sexo feminino) } \\
5.149 \mathrm{R} \\
4.702 \mathrm{D}\end{array}$ & $\begin{array}{l}\text { Entre as trabalhadoras do turno rotativo, a prevalência de sobre- } \\
\text { peso }(18,6 \%) \text { e de obesidade }(7,4 \%) \text { aumentou proporcional- } \\
\text { mente ao tempo de trabalho em turnos }(p<0,001) \text {. }\end{array}$ \\
\hline $\begin{array}{l}\text { Bogossian et al. } 2012 \\
\text { Austrália }\end{array}$ & Coorte & $\begin{array}{l}4996^{*} \\
\text { (sexo feminino) }\end{array}$ & $\begin{array}{l}61,87 \% \text { da amostra apresentaram sobrepeso ou obesidade de } \\
\left.\text { (IMC } \geq 25 \mathrm{~kg} / \mathrm{m}^{2}\right) .\end{array}$ \\
\hline $\begin{array}{l}\text { Han et al., } 2011 \\
\text { Estados Unidos }\end{array}$ & Transversal & $\begin{array}{c}2103^{*} \\
\text { (sexo feminino) }\end{array}$ & $57,3 \%$ da amostra apresentavam sobrepeso ou obesidade. \\
\hline $\begin{array}{l}\text { Zhao et al., } 2011 \\
\text { Austrália }\end{array}$ & Coorte & $\begin{array}{l}2494 \\
\text { (sexo feminino) } \\
1259 \mathrm{D} \\
1235 \mathrm{R}\end{array}$ & $\begin{array}{l}31,8 \% \text { das participantes apresentaram sobrepeso e } 26,9 \% \text {, obesi- } \\
\text { dade. Trabalhadores em turnos rotativos tinham } 1,15 \text { mais chance } \\
\text { de ter excesso de peso/obesidade que os trabalhadores do turno } \\
\text { fixo diurno. }\end{array}$ \\
\hline
\end{tabular}

Continua 
Quadro 1 (cont.)

\begin{tabular}{|c|c|c|c|}
\hline Autor(es), Ano, País & Delineamento & População & Principais resultados \\
\hline $\begin{array}{l}\text { Wong et al., } 2010 \\
\text { China }\end{array}$ & Transversal & $\begin{array}{l}378 \mathrm{R} \\
(32 \text { sexo masculino e } \\
346 \text { sexo feminino) }\end{array}$ & $\begin{array}{l}20,1 \% \text { apresentavam IMC superiores a } 23 \mathrm{~kg} / \mathrm{m}^{2} \text { o que, de acordo } \\
\text { com as faixas de peso preestabelecidas para esta população, in- } \\
\text { dica obesidade. }\end{array}$ \\
\hline $\begin{array}{l}\text { Tavares et al., } 2010 \\
\text { Brasil }\end{array}$ & Transversal & $\begin{array}{c}418^{*} \\
\text { (ambos os sexos) }\end{array}$ & $\begin{array}{l}45,3 \% \text { dos participantes apresentavam sobrepeso e } 32,9 \% \text {, obe- } \\
\text { sidade }(p<0,05) \text {. }\end{array}$ \\
\hline $\begin{array}{l}\text { Sampedro et al., } 2010 \\
\text { Espanha }\end{array}$ & Transversal & $\begin{array}{l}207 \\
35 \mathrm{~F} \\
172 \mathrm{R}\end{array}$ & $\begin{array}{l}\text { A média de IMC foi de } 23,17 \mathrm{Kg} / \mathrm{m}^{2} .19 \% \text { apresentaram sobre- } \\
\text { peso e } 4 \% \text {, obesidade. Foi encontrada uma alimentação mais sau- } \\
\text { dável no turno rotatório quando comparada ao turno fixo. }\end{array}$ \\
\hline $\begin{array}{l}\text { Botolli, Moraes \& } \\
\text { Goldmeier; } 2009 \\
\text { Brasil }\end{array}$ & Transversal & $\begin{array}{l}80 \\
(11 \text { sexo masculino e } \\
69 \text { sexo feminino })\end{array}$ & $\begin{array}{l}\text { O sobrepeso e a obesidade estavam presentes em } 56,3 \% \text { da } \\
\text { amostra, além disso, } 75 \% \text { da amostra apresentou a circunferência } \\
\text { da cintura acima dos valores limítrofes ( } \geq 94 \mathrm{~cm} \text { para homens e } \\
\geq 80 \mathrm{~cm} \text { para mulheres). }\end{array}$ \\
\hline $\begin{array}{l}\text { Miller et al., } 2007 \\
\text { Estados Unidos }\end{array}$ & Transversal & $\begin{array}{l}760^{*} \\
(61 \text { sexo masculino e } \\
699 \text { sexo feminino) }\end{array}$ & $\begin{array}{l}\text { A média de IMC foi } 27,2 \mathrm{~kg} / \mathrm{m}^{2} \text {. } \\
54 \% \text { estavam com sobrepeso ou obesos. }\end{array}$ \\
\hline Fischer et al., 2006 & Transversal & $\begin{array}{l}996 \mathrm{D} \text { e } \mathrm{N} \\
(118 \text { sexo masculino e } \\
878 \text { sexo feminino) }\end{array}$ & $\begin{array}{l}\text { Prevalência de } 21,5 \% \text { de sobrepeso }\left(25 \mathrm{Kg} / \mathrm{m}^{2} \geq \mathrm{IMC} \leq\right. \\
\left.29,9 \mathrm{Kg} / \mathrm{m}^{2}\right) \text { e de } 35,8 \% \text { de obesidade }\left(\mathrm{IMC} \geq 30 \mathrm{Kg} / \mathrm{m}^{2}\right) \\
\text { nos profissionais da área de enfermagem. }\end{array}$ \\
\hline $\begin{array}{l}\text { Ha \& Park, } 2005 \\
\text { Coreia }\end{array}$ & Transversal & $\begin{array}{l}360 \mathrm{~N} \\
(134 \text { sexo masculino e } \\
226 \text { sexo feminino })\end{array}$ & $\begin{array}{l}\text { A RCQ crescia proporcionalmente ao número de anos } \\
\text { trabalhados a noite }(p<0,05) \text {. }\end{array}$ \\
\hline $\begin{array}{l}\text { Reiners et al., } 2004 \\
\text { Brasil }\end{array}$ & Transversal & $\begin{array}{l}204^{*} \\
(20 \text { sexo masculino e } \\
152 \text { sexo feminino })\end{array}$ & $\begin{array}{l}36,6 \% \text { foram classificados com sobrepeso, } 25,6 \% \text {, com obe- } \\
\text { sidade e } 2,3 \% \text {, com obesidade mórbida. }\end{array}$ \\
\hline $\begin{array}{l}\text { Geliebter et al., } 2000 \\
\text { Estados Unidos }\end{array}$ & Transversal & $\begin{array}{l}85 \text { (ambos os sexos) } \\
36 \mathrm{D} \\
49 \mathrm{~N}\end{array}$ & $\begin{array}{l}\text { Foi verificado que o grupo noturno teve um ganho de peso } \\
\text { maior que o grupo diurno }(p=0,02) \text {. No entanto, não foram } \\
\text { encontradas diferenças significantes entre ao valores de IMC } \\
\text { de ambos os grupos. }\end{array}$ \\
\hline
\end{tabular}

$D=$ Turno diurno; $N=$ Turno noturno; $F=$ Turno fixo; $R=$ Turno rotativo; $M=$ Manhã $T=$ Tarde; $*$ turno não especificado Fonte: ScieELO e PubMed, 2014

\section{Consumo alimentar de profissionais de enfermagem}

O padrão de consumo alimentar dos trabalhadores em turnos demonstra características peculiares quando se compara trabalhadores dos turnos fixos diurno com turnos fixos noturnos e, de forma geral, pode-se dizer que ambos os turnos apresentam hábitos alimentares inadequados ${ }^{(30)}$. Porém, essa afirmação decorre de evidências limitadas na literatura, já que poucos estudos correlacionam o trabalho em turnos com a ingestão alimentar em profissionais de enfermagem ${ }^{(9,31-32)}$. O Quadro 2 mostra estudos da literatura que analisaram o consumo alimentar de profissionais da área de enfermagem.

As mudanças do consumo alimentar parecem se associar fortemente com a prática do trabalho em turnos. Ao estudar 1932 profissionais de enfermagem dos turnos diurno e noturno na Noruega ${ }^{(33)}$, observou-se uma grande ingestão de alimentos ou bebidas com cafeína, com o intuito de manter o estado de alerta. A odds ratio para esse padrão de consumo foi de 1,01 (IC de $95 \%=0,97-1,04$ ). Foi encontrado um elevado consumo de cafeína entre 80 profissionais de enfermagem do turno noturno nos Estados Unidos ${ }^{(5)}$. No Brasil ${ }^{(23)}$, também se identificou alto consumo de cafeína em amostra de profissionais de enfermagem $(70,3 \%$ dos participantes bebiam café regularmente).

Uma pesquisa que avaliou os efeitos do turno rotativo sobre o padrão alimentar de enfermeiros no Malawi ${ }^{(6)}$ encontrou, na comparação com enfermeiros do turno diurno, que profissionais do turno rotativo apresentaram menor consumo de grandes refeições por dia (2,06 grandes refeições por dia para o grupo diurno versus 1,13 para o grupo rotativo noturno, com $p<0,001)$, menor apetite e menor satisfação em comer. Todos os profissionais do turno rotativo apresentaram padrões de alimentação irregulares. Foi encontrada também uma associação significante entre a auto avaliação da saúde e o apetite, a quantidade de refeições completas e o padrão de saciedade $(p<0,05)$.

O consumo alimentar em trabalhadores noturnos também foi verificado em estudo ${ }^{(29)}$ em que os trabalhadores noturnos relataram ter um número menor de refeições diárias $(p=0,002)$, as quais eram consumidas mais tarde entre os trabalhadores, quando comparados àqueles em turnos diurnos $(p<0,00005)$. 
Com o objetivo de investigar a relação entre o trabalho noturno e os hábitos de dieta, conduziu-se um estudo ${ }^{(7)}$ com 27 profissionais de enfermagem do turno da noite na Suécia. Os participantes relataram que a fadiga e o estresse causados pelo turno de trabalho influenciaram o abandono da dieta saudável. Os indivíduos que tinham uma dieta pouco saudável à noite declararam que comiam estes alimentos ou para ficarem acordados ou porque apresentavam um desejo particular durante o turno noturno. Os voluntários também relataram um desejo por alimentos doces e por carboidratos no dia seguinte ao trabalho em um turno noturno, e descreveram também que se sentiam especialmente cansados nesse dia e, por isso, frequentemente escolhiam refeições que demandavam um preparo mais fácil e rápido.

Estudo semelhante ${ }^{(8)}$ identificou que trabalhadores do turno noturno preferiam consumir refeições frias em vez de refeições quentes $(0,76$ versus 0,23 , respectivamente com $p<0,001)$. Além disso, o tipo e a frequência das refeições foram influenciados mais significativamente $(p<0,005)$ pelo hábito e pela disponibilidade de tempo do que pelo apetite. Outro achado foi que os trabalhadores do turno diurno consumiam menos salgadinhos que os trabalhadores da noite $(0,21$ versus 0,42 respectivamente, com $p<0,05)$.
Entre os fatores postulados pela literatura como capazes de influenciar o consumo alimentar desses trabalhadores, destacam-se os fatores ambientais, o débito de sono e a dessincronização do ciclo claro-escuro, aos quais os profissionais da área de enfermagem estão submetidos ${ }^{(8,10,30)}$. Apesar de pouco explorada pela literatura, a relação da modificação dos hábitos alimentares com o trabalho em turnos parece sofrer importante influência do padrão de sono ${ }^{(34-35)}$.

Sabe-se que a diminuição do tempo de sono modula o ciclo sono-vigília e pode alterar o comportamento alimentar de diferentes maneiras ${ }^{(30)}$. Uma hipótese sugere que um maior tempo acordado implica em mais tempo disponível para comer, teoricamente facilitando as oportunidades para o consumo de lanches ou de refeições adicionais ao longo do dia ${ }^{(11,36)}$. Nesta mesma linha, estudos indicam que a restrição do sono é muito associada a irregularidades da ingestão alimentar, com grande frequência, em individuos privados de sono, de comportamentos como "beliscar" em excesso ao longo do dia e maior consumo de alimentos de alta densidade calórica ${ }^{(10,37-40)}$. Tais alterações podem ser atribuídas à resposta metabólica do organismo à privação do sono, que envolve principalmente os hormônios leptina e grelina - hormônios relacionais ao

Quadro 2 - Consumo alimentar de profissionais da área de enfermagem

\begin{tabular}{|c|c|c|c|}
\hline Autor(es), Ano, País & Delineamento & População & Principais resultados \\
\hline $\begin{array}{l}\text { Flo et al., } 2012 \\
\text { Noruega }\end{array}$ & Transversal & $\begin{array}{c}1932 \\
\text { (186 masculino e } \\
1738 \text { feminino) } \\
619 \mathrm{D} \\
1313 \mathrm{~N}\end{array}$ & $\begin{array}{l}\text { Foi observada uma alta ingestão de alimentos ou bebidas com } \\
\text { cafeína no turno da noite. }\end{array}$ \\
\hline $\begin{array}{l}\text { Geiger-Brown et al. } 2012 \\
\text { Estados Unidos }\end{array}$ & Transversal & $\begin{array}{c}80 \mathrm{~N} \\
\text { Ambos os sexos }\end{array}$ & $\begin{array}{l}\text { Consumo frequente e em grandes quantidades de cafeína para } \\
\text { manter o estado de alerta. }\end{array}$ \\
\hline $\begin{array}{l}\text { Sahu \& Dey, } 2011 \\
\text { India }\end{array}$ & Transversal & $\begin{array}{l}75 \\
\text { (sexo feminino) } \\
40 \mathrm{R} \\
35 \mathrm{D}\end{array}$ & $\begin{array}{l}\text { Profissionais de enfermagem que trabalhavam a noite fizeram um } \\
\text { número menor de refeições quentes, apresentaram menor apetite, } \\
\text { menor satisfação em comer e fizeram maior número de lanches } \\
\text { do que as trabalhadoras do turno diurno }(p<0,0005) \text {. }\end{array}$ \\
\hline $\begin{array}{l}\text { Celik et al., } 2008 \\
\text { Turquia }\end{array}$ & Transversal & $\begin{array}{c}110 \mathrm{~N} \\
\text { (sexo feminino) }\end{array}$ & $\begin{array}{l}\text { Profissionais de enfermagem que trabalhavam muitas horas a } \\
\text { noite possuíam hábitos alimentares inadequados. }\end{array}$ \\
\hline $\begin{array}{l}\text { Zererev et al., } 2005 \\
\text { Malawi }\end{array}$ & Transversal & $\begin{array}{c}24 \mathrm{R} \\
22 \mathrm{D} \\
\text { (sexo feminino) }\end{array}$ & $\begin{array}{l}\text { Grupo rotativo relatou fazer mais refeições }(p<0,001) \text { além de } \\
\text { ter os padrões de apetite e satisfação reduzidos }(p<0,01) \text { quando } \\
\text { comparados ao grupo diurno. }\end{array}$ \\
\hline $\begin{array}{l}\text { Reiners et al., } 2004 \\
\text { Brasil }\end{array}$ & Transversal & $\begin{array}{l}204^{*} \\
(20 \text { sexo masculino } \\
\text { e } 152 \text { sexo } \\
\text { feminino) }\end{array}$ & $\begin{array}{l}70,3 \% \text { dos participantes afirmaram ingerir café, } 77,9 \% \text { alegou } \\
\text { preocupar-se com a quantidade de gordura na alimentação e } 61 \% \\
\text { afirmou preocupar-se com o sal na alimentação regularmente. }\end{array}$ \\
\hline $\begin{array}{l}\text { Waterhouse et al., } 2003 \\
\text { Reino Unido }\end{array}$ & Transversal & $\begin{array}{c}50 \mathrm{D} \\
43 \mathrm{~N} \\
\text { (ambos os sexos) }\end{array}$ & $\begin{array}{l}\text { Os trabalhadores da noite relataram aumento no consumo de } \\
\text { refeições frias }(p<0,001) \text { e maior consumo de salgadinhos } \\
(p=0,01) \text {. }\end{array}$ \\
\hline
\end{tabular}

$D=$ Turno diurno; $N=$ Turno noturno; $F=$ Turno fixo; $R=$ Turno rotativo; $M=$ Manhã; $T=$ Tarde; *turno não especificado.

Fonte: ScieELO e PubMed, 2014. 
controle central da alimentação. A leptina fornece informações sobre o equilíbrio energético para o centro regulatório do cérebro e a sua liberação está associada com a promoção da saciedade ${ }^{(41-42)}$. Já a grelina tem seus níveis aumentados em períodos de jejum e atua diretamente na sensação de fome e aumento do apetite ${ }^{(43-44)}$.

De acordo com recentes investigações - em maioria realizada em ambientes laboratoriais controlados - indivíduos submetidos à privação ou restrição do sono podem apresentar dimunição nos níveis de leptina e aumento das concentrações de grelina, o que consequentemente afetaria a ingestão de alimentos $^{(10,12,45-48)}$. Recentemente, encontrou-se que trabalhadores em turnos apresentaram um padrão circadiano diferenciado na liberação desses hormônios, com importante impacto na ingestão alimentar ${ }^{(34)}$. No entanto, ainda são necessários novos estudos para melhor entendimento da ação destes hormônios em situações de déficit de sono e entre profissionais da área da Enfermagem.

\section{Hábitos de sono de profissionais de enfermagem}

Estudos recentes correlacionaram o trabalho em turnos às alterações nos hábitos de sono em profissionais da área de enfermagem, indicando que o esquema de trabalho noturno prejudica o padrão de sono ${ }^{(4,33,49)}$. O Quadro 3 mostra estudos da literatura que analisaram os hábitos de sono dos profissionais da área de enfermagem.

Um estudo transversal conduzido na Noruega ${ }^{(33)}$ encontrou que os profissionais do turno rotativo noturno apresentaram uma elevada prevalência $(37,6 \%)$ de sonolência diurna excessiva e de insônia quando comparados aos profissionais do turno diurno. Os autores ainda encontraram que a cada 50 noites trabalhadas, aumentavam em 50\% as chances de se desenvolver problemas relacionados ao sono. Estes achados também foram revelados por Hadsson \& Gustavsson ${ }^{(50)}$ que demonstraram que a qualidade do sono de indivíduos suecos reduzia à medida que os anos de trabalho noturno aumentavam. Além disso, Geiger-Brow et al. ${ }^{(5)}$ identificaram alto nível de sonolência diurna (Karolinska Sleepiness Scale (KSS) $>7$ ), escores indicando alto nível de sonolência), em $47 \%$ da amostra de 80 profissionais da área de enfermagem do turno noturno nos Estados Unidos. Um terço destes profissionais também apresentou fadiga e insônia.

Lin et al. ${ }^{(49)}$, ao avaliarem a qualidade do sono de profissionais da área de enfermagem dos turnos diurno e noturno, verificaram que os profissionais do turno noturno apresentaram uma qualidade de sono pior que a dos trabalhadores diurnos $(p<0,001)$. Os resultados deste e de outros estudos semeIhantes sugerem que o trabalho noturno promove uma piora na qualidade do sono de profissionais da área de enfermagem (Índice de Qualidade do Sono de Pittsburgh (PSQI) > 5, com escores indicando uma má qualidade do sono) ${ }^{(51-53)}$.

Uma investigação brasileira ${ }^{(4)}$ verificou que a duração do sono dos trabalhadores de enfermagem do turno noturno era, em média, 2 horas inferior à duração do sono dos enfermeiros do turno diurno $(p<0,004)$. Neste estudo foi também encontrado que o sono fracionado foi encontrado apenas nos profissionais do turno da noite. Um estudo semelhante ${ }^{(54)}$ também detectou diferenças significativas na duração do sono de profissionais de enfermagem dos turnos noturno e diurno $(p<0,001)$. Foi observado que, após uma noite de trabalho, a qualidade do sono ruim ( $p<0,007)$, além de diferenças significantes na percepção dos estados de alerta no grupo noturno $(p<0,001)$.

Uma pesquisa realizada com profissionais de enfermagem do turno fixo noturno em Taiwan ${ }^{(55)}$ revelou que $57 \%$ da amostra apresentou qualidade do sono ruim (PSQI $>5$ ) e que este resultado pode ser associado a uma piora na qualidade de vida destes profissionais como a ocorrência de distrofia pré-menstrual, acidentes de trabalho, doenças e uso de medicamentos. Analisando o nível de sonolência entre trabalhadores de enfermagem dos turnos diurno e noturno ${ }^{(29)}$, identificou-se que os trabalhadores noturnos cochilavam mais vezes $(p=0,01)$ e durante mais tempo $(p=0,05)$ durante a semana de trabalho que os trabalhadores diurnos. Outro estudo ${ }^{(56)}$ também verificou que as profissionais da área da Enfermagem que trabalhavam no turno noturno apresentaram maiores chances de insônia $(\mathrm{OR}=1.48,95 \% \mathrm{Cl}=1.10-1.99$ e fadiga crônica $(\mathrm{OR}=1.78,95 \% \mathrm{Cl}=1.02-3.11)$.

Quadro 3 - Hábitos de sono de profissionais da área de enfermagem

\begin{tabular}{|c|c|c|c|}
\hline Autor(es), Ano, País & Delineamento & População & Principais resultados \\
\hline $\begin{array}{l}\text { Øyane et al, } 2013 \\
\text { Noruega }\end{array}$ & Transversal & $\begin{array}{c}2035 \mathrm{~N} \\
\text { (sexo feminino) }\end{array}$ & $\begin{array}{l}\text { Profissionais de enfermagem com menor tempo de tra- } \\
\text { balho no turno noturno apresentaram maiores chances de } \\
\text { insônia }(\mathrm{OR}=1.48,95 \% \mathrm{Cl}=1.10-1.99) \text { e fadiga crônica } \\
(\mathrm{OR}=1.78,95 \% \mathrm{Cl}=1.02-3.11) \text { do que as trabalhadoras } \\
\text { que já tinham uma longa experiência de trabalho noturno. }\end{array}$ \\
\hline $\begin{array}{l}\text { Flo et al., } 2012 \\
\text { Noruega }\end{array}$ & Transversal & $\begin{array}{l}1932 \\
\text { (186 sexo masculino } \\
\text { e } 1738 \text { sexo } \\
\text { feminino) } \\
619 \mathrm{D} \\
1313 \mathrm{~N}\end{array}$ & $\begin{array}{l}\text { Alta prevalência }(37,6 \%) \text { de sonolência diurna excessiva e } \\
\text { insônia nos profissionais dos turnos rotativos. }\end{array}$ \\
\hline
\end{tabular}


Quadro 3 (cont.)

\begin{tabular}{|c|c|c|c|}
\hline Autor(es), Ano, País & Delineamento & População & Principais resultados \\
\hline $\begin{array}{l}\text { Geiger-Browm et al. } 2012 \\
\text { Estados Unidos }\end{array}$ & Transversal & $\begin{array}{c}80 \mathrm{~N} \\
\text { (ambos os sexos) }\end{array}$ & $\begin{array}{l}45 \% \text { apresentaram alto nível de sonolência }(\mathrm{KSS}>7) \text {, fa- } \\
\text { diga e estavam mais vulneráveis à perda de sono. }\end{array}$ \\
\hline $\begin{array}{l}\text { Lin et al., } 2012 \\
\text { Taiwan }\end{array}$ & Transversal & $\begin{array}{l}1360 \\
\text { (sexo feminino) } \\
769 \text { R } \\
591 \mathrm{D}\end{array}$ & $\begin{array}{l}\text { Os profissionais d enfermagem do turno rotativo noturno } \\
\text { apresentaram má qualidade do sono comparado aos profis- } \\
\text { sionais do turno diurno }(p<0,001) \text {. }\end{array}$ \\
\hline $\begin{array}{l}\text { Zencirci, AD; Arslan S., } 2011 \\
\text { Turquia }\end{array}$ & Transversal & $\begin{array}{c}483 \\
\text { (sexo feminino) } \\
107 \mathrm{D} \\
19 \mathrm{~N} \\
256 \mathrm{R}\end{array}$ & $\begin{array}{l}\text { Enfermeiros que trabalhavam nos turnos da manhã ou da } \\
\text { noite tiveram melhor qualidade do sono que aqueles que } \\
\text { trabalhavam no turno rotativo e diurno }(p<0,001) \text {. }\end{array}$ \\
\hline $\begin{array}{l}\text { Hsieh et al., } 2011 \\
\text { Taiwan }\end{array}$ & Transversal & $\begin{array}{l}661 \mathrm{R} \\
\text { (31 sexo masculino e } \\
630 \text { sexo feminino) }\end{array}$ & $\begin{array}{l}59 \% \text { apesentaram sono de má qualidade (PSQI>5) e in- } \\
\text { sônia. }\end{array}$ \\
\hline $\begin{array}{l}\text { Hasson, D.; Gustavsson, P. } 2010 \\
\text { Suécia }\end{array}$ & Transversal & $\begin{array}{l}2103^{*} \\
\text { (sexo feminino) }\end{array}$ & $\begin{array}{l}\text { Os resultados sugerem uma redução contínua na quali- } \\
\text { dade do sono dos profissionais de enfermagem ao longo } \\
\text { dos anos do estudo. }\end{array}$ \\
\hline $\begin{array}{l}\text { Shao et al., } 2010 \\
\text { Taiwan }\end{array}$ & Transversal & $\begin{array}{c}435 \mathrm{~F} \\
\text { (sexo feminino) }\end{array}$ & $\begin{array}{l}57 \% \text { apresentaram sono de má qualidade (PSQI }>5 \text { ). Es- } \\
\text { ses escores foram associados à piora na qualidade de vida } \\
\text { (distrofia pré-menstrual, acidentes de trabalho, doenças e } \\
\text { uso de medicação). }\end{array}$ \\
\hline $\begin{array}{l}\text { Garde et al., } 2009 \\
\text { Dinamarca }\end{array}$ & Transversal & $\begin{array}{l}166 \\
27 \mathrm{D} \\
12 \mathrm{~T} \\
47 \mathrm{~N} \\
80 \mathrm{R}\end{array}$ & $\begin{array}{l}\text { Foi observada pior qualidade de sono }(p<0,001) \text { nos enfer- } \\
\text { meiros do turno rotativo noturno comparados à qualidade } \\
\text { de sono dos profissionais de enfermagem dos turnos fixos. }\end{array}$ \\
\hline $\begin{array}{l}\text { Geliebter et al., } 2005 \\
\text { Estados Unidos }\end{array}$ & Transversal & $\begin{array}{c}85 \text { (ambos os sexos) } \\
36 \mathrm{D} \\
49 \mathrm{~N}\end{array}$ & $\begin{array}{l}\text { Trabalhadores noturnos relataram maior número }(p=0,01) \text { e } \\
\text { duração }(p=0,05) \text { de cochilos que os trabalhadores do dia. }\end{array}$ \\
\hline $\begin{array}{l}\text { Martino, } 2002 \\
\text { Brasil }\end{array}$ & Transversal & $\begin{array}{c}59 \\
(25 \text { sexo feminino e } \\
34 \text { sexo masculino) } \\
45 \mathrm{D} \\
14 \mathrm{~N}\end{array}$ & $\begin{array}{l}\text { A duração do sono noturno dos enfermeiros que trabalham } \\
\text { a noite foi, em media, } 2 \text { horas a mais que a dos enfermeiros } \\
\text { que trabalham durante o dia }(p<0,004) \text {. O sono fraciona- } \\
\text { do foi encontrado apenas no grupo noturno. }\end{array}$ \\
\hline $\begin{array}{l}\text { Fischer et al., } 2002 \\
\text { Brasil }\end{array}$ & Transversal & $\begin{array}{l}45 \\
\text { Ambos os sexos } \\
26 \mathrm{R} \\
19 \mathrm{D}\end{array}$ & $\begin{array}{l}\text { Foram detectadas diferenças significativas entre sono di- } \\
\text { urno e noturno }(p<0,001) \text {. Foi observada uma pior quali- } \\
\text { dade de sono diurno após noites de trabalho ( } p<0,007) \text {. } \\
\text { Foram encontradas diferenças significantes na percepção } \\
\text { dos estados de alerta no turno da noite }(p<0,001) \text {. }\end{array}$ \\
\hline
\end{tabular}

$D=$ Turno diurno; $N=$ Turno noturno; $F=$ Turno fixo; $R=$ Turno rotativo; $M=$ manhã; $T=$ Tarde; *turno não especificado.

Fonte: ScieELO e PubMed, 2014.

Relação entre sobrepeso, obesidade e hábitos de sono

Atualmente, é amplamente postulado pela literatura que a alteração no tempo de sono está relacionada a um descontrole no consumo alimentar e à obesidade ${ }^{(30)}$.

Simultaneamente ao aumento da obesidade divulgada em inquéritos epidemiológicos ${ }^{(20,57)}$ tem sido observado em uma redução do tempo total de sono, principalmente entre a população dos países industrializados. A duração média do sono nos Estados Unidos passou de 8-9 horas por noite em 1960 (58), para sete horas em $1995^{(59)}$. No Japão, no mesmo período a média passou de 8 horas e 13 minutos para sete horas e 32 minutos $^{(38)}$. No Brasil, um estudo realizado com uma amostra representativa populacional, identificou uma redução no tempo de sono entre os anos de 1987 e 1995, de 7.82 horas para 7,60 horas, respectivamente durante a semana; e de 9 para 8.39 horas, respectivamente nos fins de semana ${ }^{(60)}$.

Uma série de estudos transversais e prospectivos tem revelado uma correlação negativa entre a duração do sono e o índice de massa corporal (IMC) ${ }^{(24,57)}$. Esta correlação foi estabelecida em estudos com populações em diferentes estágios de vida e em diversos países da Europa, Ásia e nos Estados Unidos $^{(46,57,61-72) \text {. }}$ 
Os mecanismos pelos quais a restrição de sono pode afetar o balanço energético e levar ao excesso de peso não estão totalmente esclarecidos. No entanto, algumas hipóteses são propostas na literatura científica. Estes aspectos tratam do nível de atividade física, homeostase energética e ingestão alimentar ${ }^{(10,30,73-74)}$.

O sono está diretamente relacionado com a conservação e restauração da energia ${ }^{(75)}$. Desta forma, a diminuição do tempo de sono pode resultar na diminuição do nível de atividade física, provavelmente devido ao cansaço, em consequência do sono insuficente em termos quantitativos e qualitativos ${ }^{(61,76)}$. Assim, individuos privados do sono podem apresentar um estilo de vida mais sedentário quando comparados àqueles que possuem padrão de sono adequado(11,77). A diminuição dos níveis de atividade física somados as alterações no controle da ingestão alimentar, como o aumento do apetipe e diminuição da saciedade (fato anteriormente apresentado) podem favorecer o balanço energético positivo e favorecer o ganho de peso de forma inadequada, aumentando a prevalência de sobrepeso e obesidade ${ }^{(34)}$.

Os profissionais da Enfermagem são constantemente submetidos a rotinas de trabalho que podem prejudicar a dinâmica de sono ${ }^{(33,49-54)}$. Nesse sentido, a privação do sono, como comumente ocorre nos profissionais do turno noturno, pode ter efeitos importantes sobre o estado nutricional e a ingestão alimentar desta população(72,78).

Apesar de algumas evidências apontando os prejuízos ao padrão de sono causado pelo trabalho noturno em profissionais da área de enfermagem, não foi localizado nesta revisão nenhum estudo que relacionasse o trabalho noturno ou a privação do sono à prevalência de sobrepeso e de obesidade observada nesta população. No entanto, foram encontradas algumas evidências que sugeriram que a privação do sono parece aumentar não somente o apetite, mas também as preferências por determinados alimentos ${ }^{(30)}$. Investigação previamente cita$\mathrm{da}^{(7)}$ mostrou que o apetite por alimentos ricos em gorduras e carboidratos, principalmente doces, era maior nestes profissionais após uma noite de trabalho. De forma semelhante, foi verificada uma preferência por alimentos frios e salgadinhos no horário de trabalho dos profissionais do turno noturno ${ }^{(8)}$.
Alguns estudos também observaram um elevado consumo de cafeína durante o turno de trabalho da noite ${ }^{(5,23,33)}$. Estas evidências são bastante preocupantes, pois além dos profissionais de enfermagem com sono privado apresentarem um padrão hormonal predisponente para consumo calórico aumentado ${ }^{(30,45-48)}$, o preenchimento destas calorias tende a ser feito com alimentos de baixa qualidade nutricional ${ }^{(7-8,30)}$.

Estudos investigando a relação entre o sono e o estado nutricional, bem como o sono e o consumo alimentar destes profissionais são escassos. E, além disso, não foi encontrada nenhuma investigação que abrangesse simultaneamente a provável relação entre o sono, o consumo alimentar e o estado nutricional de profissionais da área de enfermagem.

\section{DISCUSSÃO}

A análise realizada constatou que a prevalência de sobrepeso e obesidade encontra-se elevada entre os profissionais da área da Enfermagem, o que provavelmente ocorre devido a exposição destes indivíduos a diversos fatores de risco para desordens nutricionais, como uma pior qualidade do sono. Além disso, os estudos relacionados aos hábitos de ingestão alimentar dos profissionais da Enfermagem mostraram, em geral, que este tópico merece atenção especial, pois o trabalho noturno faz com que os hábitos alimentares dos profissionais em questão sejam modificados de forma negativa com a finalidade de uma adaptação a esta rotina de trabalho.

De acordo com os estudos recentes que investigaram o padrão de sono das equipes de enfermagem, é possível julgar que os prejuízos na dinâmica do sono podem estar relacionados com a alta prevalência de sobrepeso e obesidade. Por meio destas pesquisas é possível supor que a interrupção ou diminuição do padrão de sono noturno pode influenciar de modo negativo a ingestão alimentar e, consequentemente, estado nutricional destes indivíduos.

Sugere-se, portanto, estudos futuros que possam investigar melhor a amplitude da influência dos hábitos de sono sobre a ingestão alimentar e o estado nutricional nos profissionais da área de enfermagem, assim como suas causas e consequências na saúde em curto e em longo prazo.

\section{REFERÊNCIAS}

1. Fischer FM, Borges FN, Rotenberg L, Nagai R, Soares NS, Rosa PL, et al. Work ability of health care of shift workers: what matters? Chronobiol Inter. 2006;23(6):1165-79.

2. Barboza JIRA, Moraes EL, Pereira EA, Reimão RNAA. Avaliação do padrão de sono dos profissionais de enfermagem dos plantões noturnos em Unidades de Terapia Intensiva. Einstein (São Paulo) [Internet]. 2008 [acesso em 01 de novembro de 2012];6(3):298-330. Disponível em: http://bases. bireme.br/cgi-bin/wxislind.exe/iah/online/?lsisScript = iah/ iah. $x$ is \&src $=$ google\&base $=$ LILACS\&lang $=p \&$ nextAction $=$ Ink\&exprSearch $=516945$ \&indexSearch $=$ ID

3. Maynardes DCD, Sarquis LMM, Kirchhof ALC. Trabalho noturno e morbidades de trabalhadores de Enfermagem. Cogitare Enferm. 2009;14(4):703-8.

4. Martino MMF. Estudo comparativo de padrões de sono em trabalhadores de enfermagem dos turnos diurno e noturno. Rev Panam salud Pública [Internet]. 2002 [acesso em 01 de novembro de 2012];12(2):95-100. Disponível em: http://www.scielosp.org/scielo.php?script=sci_arttex t\&pid $=$ S1020-49892002000800004

5. Geiger-Brown J, Rogers VE, Han K, Trinkoff A, Bausell $R B$, Scharf SM. Occupational Screening for sleep disorders in 12-h shift nurses using the Berlin Questionnaire. Sleep Breath [Internet]. 2013 [cited 2012 November 
01];17(1):381-8. Available from: http://www.ncbi.nlm. nih.gov/pubmed/22535196

6. Zverev YP. The impact of rotating shift work on eating patterns and self-reported health of nurses in Malawi. Malawi Med Journal [Internet]. 2005 [cited 2012 November 01];16(2):37-9. Available from: file:///C:/Users/Cliente/ Downloads/10857-25493-1-PB.pdf

7. Persson $M$, Martensson J. Situation influencing habits in diet and exercise among nurses working. J Nurs Manag [Internet]. 2006 [cited 2012 November 01];14(5):414-23. Available from: http://www.ncbi.nlm.nih.gov/pubmed/16787477

8. Waterhouse J, Buckley P, Edwards B, Reilly T. Measurement of, and some reasons for, differences in eating habits between night and day workers. Chronobiol Int [Internet]. 2003 [cited 2012 November 01];20(6):1075-92. Available from: http://www.ncbi.nlm.nih.gov/pubmed/14680144

9. Wong $\mathrm{H}$, Wong MC, Wong SY, Lee A. The association between shift duty and abnormal eating behavior among nurses working in a major hospital: a cross-sectional study. Int J Nurs Stud [Internet]. 2010 [cited 2012 November 01];47(8):1021-7. Available from: http://www.ncbi. nlm.nih.gov/pubmed/20116059

10. Spiegel K, Leproult R, L'hermite-Baleriaux M, Copinschi G, Penev PD, Van Cauter E. Leptin levels are dependent on sleep duration: relationships with sympathovagal balance, carbohydrate regulation, cortisol, and thyrotropin. J Clin Endocrinol Metab [Internet]. 2004 [cited 2012 November 01];89(11):5762-71. Available from: http://www. ncbi.nlm.nih.gov/pubmed/15531540

11. Knutson KL, Spiegel K, Penev P, Van Cauter E. The metabolic consequences of sleep deprivation. Sleep Med Rev [Internet]. 2007 [cited 2012 November 01];11(3):163-78. Available from: http://www.ncbi.nlm.nih.gov/pubmed/17442599

12. Taheri $\mathrm{S}$. The link between short sleep duration and obesity: we should recommend more sleep to prevent obesity. Arch Dis Child [Internet]. 2006 [cited 2012 November 01];91(11):881-4. Available from: http://www.ncbi.nlm. nih.gov/pubmed/17056861

13. Crispim CA, Zimberg IZ, Datillo M, Padilha HG, Tufik $S$, Mello MT. Trabalho em turnos e aspectos nutricionais: uma revisão. Nutrire Rev Soc Bras Aliment Nutr [Internet]. 2009 [cited 2012 November 01];34(2)213-27. Disponível em: http://www.revistanutrire.org.br/files/v34n2/ v34n2a15.pdf

14. Mendes KDS, Silveira RCCP, Galvão CM. Revisão integrativa: método de pesquisa para a incorporação de evidências na saúde e na enfermagem. Texto \& Contexto Enferm [Internet]. 2008 [acesso em 01 de novembro de 2012];17(4):758-64. Disponível em: http:// www.scielo.br/scielo.php?script $=$ sci_arttext\&pid $=$ S0104-07072008000400018

15. Barbosa LR, Melo MRAC. Relações entre qualidade da assistência de enfermagem: revisão integrativa da literatura. Rev Bras Enferm [Internet]. 2008 [acesso em 01 de novembro de 2012];61(3):366-70. Disponível em: http://www.scielo.br/scielo.php?script=sci arttext\&pid $=$ S0034-71672008000300015

16. Beyea S, Nicoll LH. Writing an integrative review. AORN J [Internet]. 1998 [cited 2012 November 01];67(4):877-80.
Available from: http://www.ncbi.nlm.nih.gov/pubmed/96 16108

17. Laupacis A, Wells G, Richardson WS, Tugwell G. Users' $^{\prime}$ guides to the medical literature. V. How to use an article about prognosis. Evidence-based Medicine Working Group. JAMA [Internet]. 1994 [cited 2012 November 01];20(272):234-7. Available from: http://www.ncbi.nlm. nih.gov/pubmed/8022043

18. Higgins JP, Green S, editors. Cochrane Handbook of Systematic Reviews of Interventions Version 5.0.2 [Internet] [SI]: Cochane collaboration; 2009 [updated 2009 September 2009; cited 2012 November 01]. Available from: http://handbook.cochrane.org/

19. Bogossian FE, Hepworth J, Leong GM, Flaws DF, Gibbons $\mathrm{KS}$, Benefer CA, et al. A cross-sectional analysis of patterns of obesity in a cohort of working nurses of midwives in Australia, New Zealand and the United Kingdom. Int J Nurs Stud [Internet]. 2012 [cited 2012 November 01];49(6):727-38. Available from: http://www.ncbi.nlm. nih.gov/pubmed/22307023

20. Ministério do Planejamento, Orçamento e Gestão (BR). Pesquisa de Orçamentos Familiares 2008-2009: antropometria e estado nutricional de crianças, adolescentes e adultos no Brasil [Internet]. Rio de Janeiro (RJ): IBGE; 2010 [cited 2012 November 01]. Disponível em: http:// www.ibge.gov.br/home/estatistica/populacao/condicaodevida/pof/2008_2009_encaa/pof_20082009_encaa.pdf

21. Botolli C, Moraes MA, Goldmeier S. Fatores de risco cardiovasculares em trabalhadores de enfermagem em um centro de referência no sul do Brasil. Cienc Enferm [Internet]. 2009 [acesso em 01 de novembro de 2012];15(3):101-9. Disponível em: http://www.scielo.cl/scielo.php?pid=S 0717-95532009000300011\&script = sci_arttext

22. Tavares DMS, Reis NA, Dias FA, Lopes FAM. Diabetes Mellitus: fatores de risco, ocorrência e cuidados entre trabalhadores de enfermagem. Acta Paul Enferm [Internet]. 2010 [acesso em 01 de novembro de 2012];23(5):671-6. Disponível em: http://www.scielo.br/pdf/ape/v23n5/14.pdf

23. Reiners AAO, Costa ALRC, Arruda ALG, Costa LMFC, Nogueira MS. Hipertensão arterial: perfil de saúde dos trabalhadores de enfermagem de um hospital universitário. Texto \& Contexto Enferm [Internet]. 2004 [acesso em 01 de novembro de 2012];13(1):41-9. Disponível em: http:// www.redalyc.org/articulo.oa? id $=71413107$

24. Kim MJ, Son $\mathrm{KH}$, Park HY, Choi DJ, Yoon $\mathrm{CH}$, Lee HY, et al. Association between shift work and obesity among female nurses: Korean Nurses' Survey. BMC Public Health [Internet]. 2013 [cited 2012 November 01];13(1):1204. Available from: http://connection.ebscohost.com/c/articles/93622557/association-between-shift-work-obesityamong-female-nurses-korean-nurses-survey

25. Ha M, Park J. Shift work and metabolic risk of cardiovascular disease. J Occup Health [Internet]. 2005 [cited 2012 November 01];47(2):89-95. Available from: http://www. ncbi.nlm.nih.gov/pubmed/15824472

26. Sámpedro ED, Maza RL, Puente MG. Hábitos de alimentación y actividad física según la turnicidad de los trabajadores de un hospital. Enferm Clín. 2010;20(4):229-35.

27. Han K, Trinkoff AM, Storr CL, Geiger-Brown J. Job 
stress and work schedules in relation to nurse obesity. J Nurs Adm [Internet]. 2011 [cited 2012 November 01];41(11):488-95. Available from: http://www.ncbi.nlm. nih.gov/pubmed/22033319

28. Celik S, Veren F, Ocakci A. Gastrointestinal complaints related to eating and drinking habits and work life and intensive care nurses in Zonguldak, Turkey. Dimens Crit Care Nurs [Internet]. 2008 [cited 2012 November 01];27(4):173-9. Available from: http://www.ncbi.nlm. nih.gov/pubmed/18580285

29. Geliebter A, Gluck ME, Tanowitz M, Aronoff NJ, Zammit GK. Work-shift period and weight change. Nutrition [Internet]. 2000 [cited 2012 November 0116(1):27-9. Available from: http://www.ncbi.nlm.nih.gov/pubmed/10674231

30. Crispim CA, Zalcman I, Dáttilo M, Padilha HG, Tufik S, Mello MT. Relação entre sono e obesidade: uma revisão da Literatura. Arq Bras Endocrinol Metab [Internet]. 2007 [acesso em 01 de novembro de 2012];51(7):1041-9. Disponível em: http://www.scielo.br/scielo.php?pid = S0004$-27302007000700004 \&$ script $=$ sci_arttext

31. Zhao I, Turner C, Bogossian F. The association between shift work, body mass index and low back pain in a cohort of nurses and midwives. J Occup Envirol Med. 2011;53(2):153-8.

32. Miller SK, Alpert PT, Cross CL. Overweight and obesity in nurses, advanced practice nurses, and nurse educators. J Am Acad Nurse Pract [Internet]. 2008 [cited 2012 November 01];20(5):259-65. Available from: http://www. ncbi.nlm.nih.gov/pubmed/18460166

33. Flo E, Pallesen S, Mageroy N, Moen BE, Gronli J, Nordhus $\mathrm{IH}$, et al. Shift work disorders in nurses: assessment, prevalence and related health problems. Plos ONE. 2012;7(4):e33981.

34. Crispim CA, Waterhouse J, Dâmaso AR, Zimberg IZ, Padilha HG, Oyama LM, et al. Hormonal appetite control is altered by shift work: a preliminary study. Metabolism. 2011;60(12):1726-35.

35. Zimberg IZ, Fernandes Júnior SA, Crispim CA, Tufik S, Mello MT. Metabolic impact of shift work. Work [Internet]. 2012 [cited 2012 November 01];41(S1):s4376-83. Available from: http://www.cepebr.org/upload/arquivo/\%7B72C622D5B277-4380-A9CD-63FC37CEEAE0\%7D_2012\%20Zimberg\%20Work.pdf

36. Hursel R, Rutters F, Gonnissen HK, Martens EA, Westerterp-Plantenga MS. Effects of sleep fragmentation in healthy men on energy expenditure, substrate oxidation, physical activity, and exhaustion measured over $48 \mathrm{~h}$ in a respiratory chamber. Am J Clin Nutr. 2011;94(3):804-8.

37. Buxton OM, Quintiliani LM, Yang $M H$, Ebbeling $C B$, Stoddard AM, et al. Association of sleep adequacy with more healthful food choices and positive workplace experiences among motor freight workers. Am J Public Health. 2009;99(Suppl. 3):s636-43.

38. Imaki M, Hatanaka Y, Ogawa Y, Yoshida Y, Tanada S. An epidemiological study on relationship between the hours of sleep and life style factors in Japanese factory workers. J Physiol Anthropol Appl Human Sci. 2002;21(2):115-20.

39. Ohida T, Kamal AM, Uchiyama M, Kim K, Takemura S,
Sone $\mathrm{T}$, et al. The influence of lifestyle and health status factors on sleep loss among the Japanese general population. Sleep. 2001;24(3):333-8.

40. Sahu S, Dey M. Changes in food intake pattern of nurses working in rapidly rotating shift. Al Ameen J Med Sci [Internet]. 2011 [cited 2012 November 01];4(1):14-22. Available from: http://connection.ebscohost.com/c/articles/57210267/changes-food-intake-pattern-nurses-working-rapidly-rotating-shift

41. Ahima RS, Prabakaran D, Mantzoros C, Qu D, Lowell B, Maratos-Flier $\mathrm{E}$, et al. Role of leptin in the neuroendocrine response to fasting. Nature. 1996;382(6588):250-2.

42. Elefteriou F, Ahn JD, Takeda S, Starbuck M, Yang X, Liu $X$, et al. Leptin regulation of bone resorption by the sympathetic nervous system and CART. Nature. 2005;434(7032):514-20.

43. Bodosi B, Gardi J, Hajdu I, Szentirmai E, Obal F Jr, Krueger JM. Rhythms of ghrelin, leptin, and sleep in rats: effects of the normal diurnal cycle, restricted feeding, and sleep deprivation. Am J Physiol Regul Integr Comp Physiol. 2004;287(5):R1071-9.

44. Lennernas MAC, Akersted T, Hagman U, Bruce A, Hambraeus L. A new approach for evaluation of meal quality and meal patterns. J Human Nutr Diet. 1993;6(3):261-73.

45. Schmid SM, Hallschmid M, Jauch-Chara K, Born J, Schultes $B$. A single night of sleep deprivation increases ghrelin levels and feelings of hunger in normal-weight healthy men. J Sleep Res. 2008;17(3):331-4.

46. Chaput JP, Despres JP, Bouchard C, Tremblay A. Short sleep duration is associated with reduced leptin levels and increased adiposity: results from the Quebec family study. Obesity (Silver Spring). 2007; 15(1):253-61.

47. Spiegel K, Tasali E, Penev P, Van Cauter E. Brief communication: sleep curtailment in healthy young men is associated with decreased leptin levels, elevated ghrelin levels, and increased hunger and appetite. Ann Intern Med. 2004; 141(11):846-50.

48. Mullington JM, Chan JL, Van Dongen HP, Szuba MP, Samaras J, Price NJ, et al. Sleep loss reduces diurnal rhythm amplitude of leptin in healthy men. J Neuroendocrinol. 2003;15(9):851-4.

49. Lin PC, Chen $\mathrm{CH}$, Pan $\mathrm{SM}$, Pan $\mathrm{CH}$, Chen $\mathrm{YM}$, Hung $\mathrm{HC}$, et al. Atypical work schedules are associated with poor sleep quality and mental health in Taiwan female nurses. Int Arch Occup Environ Health. 2012;85(8):877-84.

50. Hasson D, Gustavsson P. Declining sleep quality among nurses: a population-based four-year longitudinal study on the transition from nursing education to working life. PLos ONE. 2010;5(12): e14265.

51. Hsieh ML, Li YM, Chang ET, Lai HL, Wang WH, Wang SC. Sleep disorder in Taiwanese nurses: a random sample survey. Nursing Health Sci. 2011;13(4):468-74.

52. Zencirci AD, Arslan S. Morning-evenig type and burnout level as factors influencing sleep quality of shift nurses: a questionnaire study. Croat Med J. 2011;52(4):527-37.

53. Garde AH, Hansen AM, Hansen J. Sleep length and quality, sleepiness and urinary melatonin among healthy Danish nurses with shift work during work leisure time. Int 
Arch Occup Environ Health. 2009; 82(10):1219-28.

54. Fischer FM, Teixeira LR, Borges FNS, Gonçalves MBL, Ferreira RM. Percepção de sono: duração, qualidade e alerta em profissionais da área de enfermagem. Cad Saúde Pública. 2002;18(5):1261-9.

55. Shao MF, Chou YC, Yeh MY, Tzeng WC. Sleep quality and quality of life in female shift-working nurses. J Adv Nurs. 2010;66(7):1565-72.

56. Oyane NMF, Pallesen S, Moen BE, Akerstedt T, Bjorvatn $B$. Associations between night work and anxiety, depression, insomnia, sleepiness and fatigue in a sample of Norwegian nurses. PLos ONE. 2013; 8(8):e70228.

57. Gangwisch JE, Malaspina D, Boden-Albala B, Heymsfield SB. Inadequate sleep as a risk factor for obesity: analyses of the NHANES I. Sleep. 2005;28(10):1289-96.

58. Kripke DF, Simons RN, Garfinkel L, Hammond EC. Short and long sleep and sleeping pills. Is increased mortality associated? Arch Gen Psychiatry. 1979;36(1):103-16.

59. Gallup. Sleep in America. Princeton (NJ): The Gallup Organization, National Sleep Foundation; 1995.p.1-78.

60. Pires ML, Benedito-Silva AA, Mello MT, Pompeia SG, Tufik S. Sleep habits and complaints of adults in the city of São Paulo, Brazil, in 1987 and 1995. Braz J Med Biol Res. 2007;40(11):1505-15.

61. Patel SR, Hu FB. Short sleep duration and weight gain: a systematic review. Obesity (Silver Spring). 2008;16(3):643-53.

62. Vioque J, Torres A, Quiles J. Time spent watching television, sleep duration and obesity in adults living in Valencia, Spain. Int J Obes Relat Metab Disord [Internet]. 2000 [cited 2012 November 01];24(12):1683-8. Available from: http://www.ncbi.nlm.nih.gov/pubmed/11126224

63. Cournot M, Ruidavets JB, Marquie JC, Esquirol Y, Baracat $B$, Ferrieres J. Environmental factors associated with body mass index in a population of Southern France. Eur J Cardiovasc Prev Rehabil. 2004;11(4):291-7.

64. Shigeta $H$, Shigeta $M$, Nakazawa A, Nakamura N, Yoshikawa T. Lifestyle, obesity, and insulin resistance. Diabetes Care. 2001;24(3):608.

65. Vorona RD, Winn MP, Babineau TW, Eng BP, Feldman $\mathrm{HR}$, Ware JC. Overweight and obese patients in a primary care population report less sleep than patients with a normal body mass index. Arch Intern Med [Internet]. 2005 [cited 2012 November 01];165(1):25-30. Available from: http://www.ncbi.nlm.nih.gov/pubmed/15642870

66. Singh M, Drake CL, Roehrs T, Hudgel DW, Roth T. The association between obesity and short sleep duration: a population-based study. J Clin Sleep Med. 2005;1(4):357-63.
67. Padez C, Mourão I, Moreira P, Rosado V. Prevalence and risk factors for overweight and obesity in Portuguese children. Acta Paediatr. 2005;94(11):1550-7.

68. Von Kries R, Toschke AM, Wurmser H, Sauerwald T, Koletzko $B$. Reduced risk for overweight and obesity in 5and 6-y-old children by duration of sleep - a cross-sectional study. Int J Obes Relat Metab Disord [Internet]. 2002 [cited 2012 November 01];26(5):710-6. Available from: http://www.ncbi.nlm.nih.gov/pubmed/12032757

69. Sekine M, Yamagami T, Handa K, Saito T, Nanri S, Kawaminami $K$, et al. A dose-response relationship between short sleeping hours and childhood obesity: results of the Toyama Birth Cohort Study. Child Care Health Dev. 2002;28(2):163-70.

70. Chen MY, Wang EK, Jeng YJ. Adequate sleep among adolescents is positively associated with health status and healthrelated behaviors. BMC Public Health [Internet]. 2006 [cited 2012 November 01];6(59):1-15. Available from: http:// www.ncbi.nlm.nih.gov/pmc/articles/PMC1447528/

71. Knutson KL. Sex differences in the association between sleep and body mass index in adolescents. J Pediatr. 2005;147(6):830-4.

72. Kohatsu ND, Tsai R, Young T, Vangilder R, Burmeister LF, Stromquist AM, et al. Sleep duration and body mass index in a rural population. Arch Intern Med. 2006;166(16):1701-5.

73. Morselli LL, Guyon A, Spiegel K. Sleep and metabolic function. Pflugers Arch. 2012;463(1):139-60.

74. Crispim CA, Zalcman I, Dáttilo M, Padilha HP, Edwards $B$, Waterhouse J, et al. The influence of sleep and sleep loss upon food intake and metabolism. Nutr Res Rev. 2007;20(2):195-212.

75. Carskadon MA, Dement W. Normal human sleep: an overview. In: Kryger, MH, Roth T, Dement W. Principles and practice of sleep medicine. 4. ed. Philadelphia (US): WB Saunders; 2005:475-81.

76. Dinges DF, Pack F, Williams K, Gillen KA, Powel JW, Ott $\mathrm{GE}$, et al. Cumulative sleepiness, mood disturbance, and psychomotor vigilance performance decrements during a week of sleep restricted to 4-5 hours per night. Sleep. 1997;20(4):267-77.

77. Atkinson G, Fullick S, Grindey C, Maclaren D. Exercise, energy balance and the shift worker. Sports Med. 2008;38(8):671-85.

78. Patel RS, Malhotra A, White DP, Gottlieb DJ, Hu FB. Association between reduced sleep and weight gain in women. Am J Epidemiol. 2006; 164(10):947-54. 